\title{
Development and Implementation of Software Systems for Imaging Spectroscopy
}

\author{
Joseph W. Boardman \\ Analytical Imaging and Geophysics, LLC \\ Boulder, CO, USA \\ boardman@aigllc.com \\ Roger N. Clark \\ Denver Spectroscopy Lab \\ U. S. Geological Survey \\ Denver, CO, USA \\ rclark@usgs.gov \\ Alan S. Mazer \\ Jet Propulsion Laboratory \\ California Institute of Technology \\ Pasadena, CA, USA \\ alan@judy.jpl.nasa.gov
}

\author{
Larry L. Biehl \\ Information Technology at Purdue (ITaP) \\ Purdue University \\ West Lafayette, IN, USA \\ biehl@purdue.edu
}

Fred A Kruse

Horizon GeoImaging, LLC

Frisco, CO, USA

kruse@hgimaging.com

\author{
James Torson \\ Astrogeology Research Program (retired) \\ U. S. Geological Survey \\ Flagstaff, AZ, USA \\ jim.torson@,computer.org
}

\author{
Karl Staenz \\ Canada Centre for Remote Sensing \\ Natural Resources Canada \\ Ottawa, ON, Canada \\ Karl.Staenz@CCRS.NRCan.gc.ca
}

\begin{abstract}
Specialized software systems have played a crucial role throughout the twenty-five year course of the development of the new technology of imaging spectroscopy, or hyperspectral remote sensing. By their very nature, hyperspectral data place unique and demanding requirements on the computer software used to visualize, analyze, process and interpret them. Often described as a marriage of the two technologies of reflectance spectroscopy and airborne/spaceborne remote sensing, imaging spectroscopy, in fact, produces data sets with unique qualities, unlike previous remote sensing or spectrometer data. Because of these unique spatial and spectral properties hyperspectral data are not readily processed or exploited with legacy software systems inherited from either of the two parent fields of study. This paper provides brief reviews of seven important software systems developed specifically for imaging spectroscopy.
\end{abstract}

Keywords-hyperspectral; imaging spectroscopy; software

\section{INTRODUCTION}

This paper reviews some of the history of the numerous software systems developed for hyperspectral data analysis. The software tools span the realm of commercial, government and academic developers. The systems range from specialized software, created for specific systems or implementing specialized algorithms, to general-use platforms that seek to serve many tasks for multiple data sources. We describe systems that are actively being updated and distributed as well as historically important legacy systems that are no longer in development or supported. The common thread of all the reviewed software systems is their contribution to unlocking the incredible power of quantitative remote sensing via imaging spectroscopy. The systems, as diverse as their various authors, all illustrate the information-rich nature of hyperspectral data and the fact that we do not yet fully understand and exploit this powerful remote sensing technology.

Software systems for visualization and interpretation of imaging spectroscopy data have been in development for nearly thirty years. The first airborne sensors developed at JPL (AIS) and Geophysical and Environmental Research (GERIS) were flying and collecting data in the early 1980's. Existing image processing packages could not directly handle the many spectral bands of these systems nor could they easily exploit the important spectral information in the data. Early on it was clear that specialized software was required. Throughout the history of imaging spectroscopy there has been a symbiotic relationship between the developers of hardware systems and software systems, each being forced into improving their product as the other made progress. The software packages represented in this review span the entire history of this 
process. In rough chronological order we include: SPAM (JPL); ISIS (USGS Flagstaff); Tetracorder (USGS Denver); SIPS (CU/CSES); MULTISPEC (Purdue); ENVI (BSC/AIG/RSI); and ISDAS (CCRS). While this is in no way a comprehensive list of all imaging spectroscopy software, it is illustrative of the diversity and development history of much of the industry.

\section{IMAGING SPECTROSCOPY SOFTWARE SYSTEMS}

\section{A. SPAM-SPectral Analysis Manager}

Spam evolved out of a simple plotting program used on Airborne Imaging Spectrometer data back in 1983 [1][2]. JPL had just received one of the first Unix workstations from the new Sun Microsystems, connected it to a Rastertek frame buffer, and tried it out using some hastily-written spectral data plotting. From there it just grew.

Compared to the packages that came later, Spam wasn't very feature-rich. It was limited to 8-bit data and provided very little image manipulation. User interfaces back then were still command-line driven. Hyperspectral image data truly stretched the capabilities of the 68000 Sun processor.

What enabled multispectral data analysis in that environment was the concept of binarily encoding image spectra, with each spectral channel encoded as a 1 or a 0 , based on whether the DN value was above or below the spectral mean. This approach was relatively fast on the processor, and less sensitive to albedo variations than other encodings. Two spectra could be compared by simply comparing their respective bit strings, evaluating the bit differences, and comparing the count against a user-specified threshold. Moreover, the encoding for a 32-channel spectrum (although 128-channel spectra could also be used) could be represented in the limited memory with just 4 bytes. Binary encoding enabled a variety of additional features beyond simple spectral matching, including automated spectral clustering, spectral identification against encoded spectral libraries, and the use of Hamming histograms to evaluate homogeneity of regions.

Other features found their way into Spam as well, including spectrum-smoothing filters, and image and spectrum arithmetic using user-entered algebraic expressions. Spectral "movies" featured tightly timed, fast display of image bands, a real trick on the old hardware available. Spectra could be printed on a dot-matrix printer. Mixture analysis, using a linear additive model, was a late addition. Finally, Spam provided not only laboratory spectra for use in spectral identification, but also user libraries, which allowed users to input their own spectra using a tablet and to save spectra plotted by Spam.

Spam wasn't used much past the late 1980's. Although Spam was ported to VAX/VMS, and attempts were made to extend it to AVIRIS data, other software was by then more popular. Parts of it have lived on, however. Some of the "virtual frame buffer" code written for Spam is still being used on other NASA missions.

\section{B. ISIS-Integrated Software for Imagers and Spectrometers}

Development of the Integrated Software for Imagers and Spectrometers (ISIS 2.0) began at the U. S. Geological Survey in 1989 and replaced an earlier prototype version [3]. ISIS was initially used for data from the Near Infrared Mapping Spectrometer (NIMS) on the Galileo Jupiter spacecraft, but it is a general-purpose software system that has been used for a number of planetary and terrestrial missions, e.g. AVIRIS. ISIS 2.0 was initially developed in the VAX/VMS environment and is implemented in a combination of the Fortran and $\mathrm{C}$ languages. The text-based user interface for the non-interactive processing programs uses the Transportable Applications Executive (TAE) developed by the Goddard Space Flight Center. The large collection of application programs includes such categories as data translators, geometric projection (a particular strength of ISIS), instrument calibration, spatial/spectral filters, statistics, arithmetic operations, and a variety of mission-specific applications. The basic data file format used by ISIS is an early version of the NASA Planetary Data System format. The programming environment supports image data arrays with up to six dimensions, but most applications use three or four dimensions. In addition to the primary image data array, the ISIS file format supports supplemental "suffix" data that extend any of the first three dimensions. The most commonly used are "backplanes" that extend the wavelength (band) dimension and have been used for data such as latitude/longitude, incidence/phase/emission angles, region-of-interest bitplanes, etc. Supported pixel formats include 32-bit floating point and 16 or 8-bit scaled integer. The 16 and 8-bit formats include base/multiplier values (stored in the image file header) that are automatically applied so that users and application programs see only the corresponding floating point values that are being represented. ISIS also supports five types of "special" pixel values: Null, High/Low Instrument Saturation, and High/Low Representation Saturation. Also included is a comprehensive processing history facility (recorded in each image data file and also in a separate session log file) and support for spectral library files and table files, which can be used for storing an average spectrum for a region-of-interest.

ISIS 2.0 included an interactive display program called QL3, which made use of the International Imaging Systems IVAS image display device and the VAXstation GPX bit-map graphics display [4]. In addition to the basic QL3 display functions, the user could also run concurrent application processes to compute and plot an average spectrum for a spatial region, plot selected laboratory spectra, identify spatial pixels that match an average spectrum, plot intensity profiles, or plot two-dimensional histograms of spatial regions for two selected wavelength bands.

In 1993, ISIS 2.1 was produced by porting ISIS 2.0 to the Unix environment. Currently supported platforms include PC/Linux, Sun/Solaris, and Mac/OS X. ISIS 2.1 includes the ability to run applications from the operating system command line or in a scripting language such as PERL. The interactive hyperspectral visualization program is called "cv" (Cube Visualization), which is implemented using the IDL language. The interactive capabilities include functions such as displaying selected spatial-spatial or spatial-spectral slices 
through the cube, "movie" display of different slices, displaying backplane images as well as "core" plane images, reporting cursor coordinates and associated pixel values, zoom and roam of displayed images, plotting single spectra and average spectra at selected spatial locations, plotting spatial profiles, hard-copy output, etc.

In recent years, development of ISIS 3.0 was begun. This is a completely new software system that is implemented in the $\mathrm{C}++$ language and uses a new data file format and a new user interface [5]. The initial development has concentrated mostly on providing geometry processing, but additional hyperspectral functionality is planned for the future.

\section{TETRACORDER}

Over the past two decades scientists at the U. S. Geological Survey developed a software system that takes an explicitly spectroscopic approach to "hyperspectral" analysis [6, and references therein] This system, called Tetracorder, is an expert system where multiple algorithms are applied to analyze a spectrum. The results of those algorithms can be tested and are compared. The system ranks the results of the algorithms and selects the best results as the answer. Each spectrum is partitioned into diagnostic wavelength regions and the algorithms are tested on different spectral regions. This enables the spectral properties from multiple materials to be analyzed and identified without the need for unmixing. The identification step appears to be a unique aspect of the Tetracorder approach not present in other published algorithms to date.

The success of the Tetracorder system is illustrated by its use on divergent environments where a single expert system command set has been used to map compounds, ranging from minerals in different geologic settings, vegetation, including species and age, water and contaminants in water, environmental materials, to the compounds and minerals in the World Trade Center disaster [7, 6 and references therein]. While the Tetracorder system uses multiple algorithms, its primary algorithm is a modified least squares spectral feature matching algorithm based on continuum removed spectral features. Other algorithms used include spectral slope, continuum level assessment, and absorption edge position.

The expertise in the system has been derived from scientific knowledge of spectral identification. The expert system rules are implemented in a decision tree where multiple algorithms are applied to spectral analysis, including the influences of mixtures. Additional expert rules and algorithms can be applied based on initial results and further decisions are made until spectral analysis is complete. Because certain spectral features are indicative of specific chemical bonds in materials, the system can accurately identify and map those materials. The decision process allows the system to compare results on a spectrum by spectrum basis; from pixel to pixel in an imaging spectroscopy data set. This decision process negates the need for thresholding by an image interpreter to distinguish between materials. Indeed, it has been shown that the thresholds needed to discriminate between different materials vary on a pixel by pixel basis due to variations in grain sizes and abundances of the materials and contaminants present [6]
The expert system in the Clark et al. (2001) World Trade Center study used over 320 reference spectra [8] with over 11,000 lines of commands. This same command set can be applied to numerous environments from geologic mapping to vegetation to disaster zones.

\section{SIPS - Spectral Image Processing System}

SIPS was an imaging spectrometer data analysis software package developed starting in 1990 at the Center for the Study of Earth from Space (CSES), University of Colorado, Boulder. This package built on concepts developed over approximately 10 years for analysis of high-spectral-resolution image data and incorporated in in-house engineering software and in software packages such as "SPAM" [2] and "ISIS" [4]. SIPS was developed under NASA funding specifically as a means of viewing analysis results from the Airborne Visible/Infrared Imaging Spectrometer [9], but was flexible enough to use with other datasets. The software was distributed world-wide and free-of-charge to over 200 organizations under a Memorandum of Understanding through the University of Colorado.

SIPS consisted of three software modules, SIPS Utilities, SIPS_View, and SIPS Analysis [10]. The SIPS Utilities allowed tape reading and disk-to-disk processing, including data formatting, correction to apparent reflectance, and cosmetic processing. SIPS_View provided interactive viewing and analysis capabilities, including interactive selection of bands for RGB composites; rapid extraction and display of spectral slices, individual spectra, or spectra from polygon regions; and the capability to visually compare and/or mathematically match image spectra to spectral libraries. SIPS Analysis was a set of programs for detailed, full-cube analysis of imaging spectrometer data, including binary encoding, the Spectral Angle Mapper (SAM), and linear spectral unmixing [11]. All of SIPS was written in a proprietary, 4GL language, the "Interactive Data Language" (IDL)[12].

\section{E. MULTISPEC}

MultiSpec is a freeware software application for analyzing multispectral and imaging spectrometer (hyperspectral) data such as LANDSAT TM, SPOT, MODIS, ASTER, IKONOS, Quickbird, ALI, Hyperion or AVIRIS data to name a few [13, 14]. MultiSpec is implemented for personal computers running the Apple Macintosh operating system (OS) including Intel, PowerPC or $680 \mathrm{x} 0$ based machines running OS7.1 thru OSX and the Microsoft Windows OS running Windows 95/98/NT/2000/ME/XP. The Windows version contains nearly all of the features that are available in the Macintosh version.

MultiSpec had its origin in the LARSYS multispectral image analysis system [15], which was one of the first remote sensing multispectral data processing systems, originally created during the 1960's. The original purpose for the development of MultiSpec, begun in 1988, was to provide an easy to use tool that could be used for teaching, research, but especially, to provide the ability for researchers to try new techniques without having to program the algorithms. It has been used in graduate level remote sensing courses to teach signal processing analysis techniques. Graduate students use it to take advantage of algorithms developed by previous students as they develop and study new algorithms. However, it has 
found additional uses in other circumstances, such as university and K-12 education, and in the government and commercial sectors. Even though the original application was for earth resources type applications, MultiSpec has also been used for analysis of multispectral medical images, microscopic images, document $\&$ art painting analyses and multispectral images of other planets.

MultiSpec recognizes many image file formats including: ArcView, ENVI, ERDAS *.LAN, ERDAS *.GIS, ERDAS Imagine, FastL7A, GAIA, TIFF/GeoTIFF, HDF-Scientific Data Model, Land Analysis System (LAS), LARSYS Multispectral Image Storage Tape (MIST), LGSOWG, MacSADIE, NetCDF, PDS, Sun "Screen Dump", TARGA, VICAR. Currently, MultiSpec can handle 8-bit and 16-bit integer data values as long as the data are not compressed; the next update for MultiSpec, though, will also be able to handle 32-bit integer, 32-bit real and 64-real data values. MultiSpec can also read and overlay ArcView shape files onto the images.

Some of the analysis features include display of selected channels of multispectral images and side-by-side channels, compute histograms, convert image data to different image file formats, unsupervised classifications (clustering), supervised classifications with a choice of classifiers including a spectralspatial classifier called ECHO[16], feature selection, feature extraction, statistics enhancement, plus others. Documentation and tutorials are available on the MultiSpec web site [13].

\section{F. ENVI - The Environment for Visualizing Imagery}

ENVI is a commercial remote sensing software system that was initially focused on providing unique and powerful tools for hyperspectral data analysis. ENVI was originally developed and owned by a five-partner Limited Liability Company in Boulder, Colorado: Better Solutions Consulting (BSC). BSC at that time had the same owners/partners as its sister company Analytical Imaging and Geophysics (AIG) which focuses on imaging spectrometry applications and research. ENVI was marketed and sold as end-user software under license from BSC by Research Systems Inc. (RSI) of Boulder, Colorado, the owners and developers of the IDL programming language, in which ENVI is written. The first version of ENVI (version 1.0) was offered for sale in February of 1994. In October, of 2000 Eastman Kodak Commercial and Government Systems bought RSI and ENVI, consolidating the ownership and technical development with the sales and marketing. Subsequently this portion of Eastman Kodak, along with RSI and ENVI, was purchased by ITT Industries.

ENVI was successful in providing a powerful, yet easy-touse, platform for exploitation of imaging spectroscopy data. AIG principals developed, then included in ENVI, tools they needed for their hyperspectral R\&D efforts. In addition, a number of third parties have created ENVI-compatible add on modules in IDL, $\mathrm{C}$ and FORTRAN that leverage the framework of ENVI for specialized commercial, government and research imaging spectroscopy applications.

An underlying theme for the exploitation tools in ENVI is the visualization, processing, understanding and analysis of the hyperspectral data in several different, yet well-linked data spaces. ENVI, of course, can view the hyperspectral images in a data cube, as well as the spectral profiles. However, beyond these common tools, ENVI introduced, or improved upon, several key visualization schemes based on 2-d and n-d scatterplots of the data in spectral space. EFFORT Spectral Polishing, the Pixel Purity Index, the n-Dimensional Visualizer and Mixture Tuned Matched Filtering were all science innovations resulting from $\mathrm{AIG} / \mathrm{BSC}$ research that form the backbone of ENVI's unique imaging spectroscopy tools. ENVI is now a more widely based image processing package, having grown beyond its early hyperspectral niche. It is a commercial product of Research Systems of Boulder, Colorado, a wholly owned subsidiary of ITT Industries.

\section{G. ISDAS - Imaging Spectrometer Data Analysis System}

ISDAS, evolved from an earlier version, has been developed since 1995 in order to expand the capability of the Canada Centre for Remote Sensing (CCRS) to analyze hyperspectral data and to establish an environment for research and development with respect to hyperspectral remote sensing. Accordingly, this system is designed to meet the following requirements: (1) a software environment which allows the rapid development of algorithms and information products, (2) a modular framework for easy addition of new tools and changes to existing tools, and (3) processing of data from any hyperspectral airborne and spaceborne sensors that acquires data in the visible near infrared (VNIR) and short-wave infrared wavelength (SWIR) regions.

ISDAS runs currently in a Red Hat Linux environment on PCs. It uses the Application Visualization System (AVS, version 5.5; [17]), a commercially available graphics and data processing software product, as the development environment. AVS is a graphical programming environment consisting of a library of data processing and visualization modules which may be connected together in networks to perform specific task. Algorithms which are not already available in AVS may be programmed in $\mathrm{C}, \mathrm{C}++$, or Fortran.

The ISDAS tools, mostly coded in $\mathrm{C}$, can be divided into the five main categories, data input/output, data visualization, data preprocessing, simulation of future sensor data, and information extraction. These tools provide the functionality to handle hyperspectral and associated ancillary data, remove sensor and calibration artifacts [18]), convert at-sensor radiance to surface reflectance [19], remove BRDF [20], interactively view and analyze data, evaluate the performance of future sensors [21]), and extract qualitative and quantitative information products [22].

ISDAS has been partially commercialized by PCI Geomatics and Vexcel Canada (now Microsoft). PCI incorporated a large portion of ISDAS tools in its general purpose system Geomatica, while Vexcel built a fast image analysis system based on parallel processing for mineral mapping purposes using ISDAS tools.

\section{SUMMARY}

Imaging spectroscopy data demand specialized software and it has been under development, and constant improvement, for more than twenty years. While existing systems are certainly diverse, robust and powerful, it is abundantly clear 
that much information remains untapped in modern hyperspectral data, awaiting new algorithms and software implementations.

\section{ACKNOWLEDGMENTS}

Each of these software systems is the product of many contributors beyond the listed authors. We readily acknowledge the various forms and sources of support and the numerous government, academic and commercial rights holders.

The SPAM binary coding concept and most of the other ideas eventually implemented in Spam came from group leader Dr. Jerry Solomon. Miki Martin and Dr. Meemong Lee provided mixture analysis and multispectral clustering algorithms, respectively.

A number of people at several organizations have contributed to the ISIS development. It should be noted that the efforts of Kris Becker, at the USGS, were particularly important in assisting with the design of ISIS 2.0, implementing much of the programming environment, many application programs, and porting ISIS to Unix.

Development of the Tetracorder-related algorithms was funded by the NASA Planetary Geology and Geophysics program and the U.S. Geological Survey Mineral Resources Program. Early developments were funded by the NASA HIRIS team.

SIPS was developed under NASA funding at the Center of the Study of Earth from Space (NAGW1601 and NAS530552). We acknowledge the support of Alex Goetz, CSES Director.

Funding for the research leading to the development of MultiSpec was provided by NASA. The Globe Program provided the support for the initial Windows versions of MultiSpec.

ENVI was initially created and owned by the five partners of BSC/AIG: Joe Boardman, Fred Kruse, Adam Lefkoff, Jim Young and Kathy Kierein Young. We acknowledge the efforts of RSI for worldwide marketing and sales of ENVI, especially the early vision of Denise Fields, who understood the value of the product.

The support of CCRS, CSA, and MDA in the development of ISDAS is gratefully acknowledged.

\section{REFERENCES}

[1] Mazer, A., M. Martin, M. Lee, J. Solomon, "Image processing software for imaging spectrometry," Imaging Spectroscopy II, Gregg Vane, Editor, Proc. SPIE 834, pp. 136-139 (1988).

[2] Mazer, A., M. Martin, M. Lee, J. Solomon, "Image processing software for imaging spectrometry data analysis," Remote Sensing of Environment, 24:201-210 (October 1987).

[3] ISIS website: http://isis.astrogeology.usgs.gov/

[4] J.M. Torson, "Interactive Image Cube Visualization and Analysis," Proceedings of the Chapel Hill Workshop on Volume Visualization, May 1989, pp. 33-38.
[5] J.A. Anderson, et al., "Modernization of the Integrated Software for Imagers and Spectrometers," abs. 2039, LPSC XXXV, 2004.

[6] Clark, R. N., G. A. Swayze, K. E. Livo, R. F. Kokaly, S. J. Sutley, J. B. Dalton, R. R. McDougal, and C. A. Gent, Imaging spectroscopy: Earth and planetary remote sensing with the USGS Tetracorder and expert systems, J. Geophys. Res., 108(E12), 5131, doi:10.1029/2002JE001847, pages 5-1 to 5-44, December, 2003a.

[7] Clark, R. N. , R. O. Green, G. A. Swayze, G. Meeker, S. Sutley, T. M. Hoefen, K. E. Livo, G. Plumlee, B. Pavri, C. Sarture, S. Wilson, P. Hageman, P. Lamothe, J. S. Vance, J. Boardman, I. Brownfield, C. Gent, L. C. Morath, J. Taggart, P. M. Theodorakos, and M. Adams, Environmental Studies of the World Trade Center area after the September 11, 2001 attack. U. S. Geological Survey, Open File Report OFR-01-0429. 2001. Web-report at: http://pubs.usgs.gov/of/2001/ofr-010429/

[8] R. N. Clark, G. A. Swayze, R. Wise, K. E. Livo, T. M. Hoefen, R. F. Kokaly, and S. J. Sutley, USGS Digital Spectral Library splib05a, USGS Open File Report 03-395, 2003 b.

[9] Kruse, F. A., Lefkoff, A. B., Boardman, J. B., Heidebrecht, K. B., Shapiro, A. T., Barloon, P. J., and Goetz, A. F. H., 1993, The Spectral Image Processing System (SIPS) - Interactive Visualization and Analysis of Imaging Spectrometer Data: Remote Sensing of Environment, Special issue on AVIRIS, May-June 1993, v. 44, pp. 145 163.

[10] Center for the Study of Earth from Space, 1992, SIPS User's Guide, The Spectral Image Processing System, v 1.1, Boulder, CO, 74 pp.

[11] Boardman, J. W., 1989, Inversion of imaging spectrometry data using singular value decomposition: in Proceedings, IGARSS A' $89,12^{\text {th }}$ Canadian Symposium on Remote Sensing, v. 4, IGARSS, Canada, pp. 2069 - 2072. (Available from the Institute of Electrical \& Electronics Engineers, Piscataway, NJ 08854).

[12] Research Systems, Inc., 1991, IDL ${ }^{\circledR}$ User's Guide Version 2.2, Boulder, $\mathrm{CO}$.

[13] MultiSpec website: http://dynamo.ecn.purdue.edu/ biehl/MultiSpec/

[14] Biehl, Larry and David Landgrebe, 2002, "MultiSpec - a tool for multispectral-hyperspectral image data analysis", Computers \& Geosciences, Vol. 28, no. 10, Dec. 2002, pp 1153-1159.

[15] Phillips,.T.L., ed., 1973. LARSYS User's Manual. Laboratory for Applications of Remote Sensing, Purdue University, West Lafayette, Indiana, (1,118 pages).

[16] Landgrebe, D.A., 1980. Development of a spectral-spatial classifier for earth observational data in Pattern Recognition 12 (3), 165-175.

[17] Upson, C., T. Faulhaber, D. Kamins, D. Laidlaw, D. Schlegel, J. Vroom, R. Gurwitz, and A. Van Dam, "The Application Visualization System: A Computational Environment for Scientific Visualization," IEEE Computer Graphics \& Applications, 9(4): 30-42, 1989.

[18] Khurshid, S., K. Staenz, L. Sun, R.A. Neville, H.P. White, A. Bannari, C.M/ Champagne, and R. Hitchcock, "Preprocessing of EO-1 Hyperion Data," Canadian Journal of Remote Sensing (in press), 2006.

[19] Staenz, K., and D. Williams, "Retrieval of Surface Reflectance From Hyperspectral Data Using a Look-Up Table Approach," Canadian Journal of Remote Sensing, 23(4): 354-368, 1997.

[20] White H.P., L. Sun, C.M. Champagne, K. Staenz, S. G. Leblanc, "BRDF Normalization of Hyperspectral Image Data," Proceedings of the International Geoscience and Remote Sensing Symposium (IGARSS'2002), Toronto, Ontario, Canada, pp. IV: 2572-2574, June 2002

[21] Sun, L., K. Staenz, R.A. Neville, and H.P. White, "Impact of Sensor Signal-to-Noise and Spectral Charactersitics on Hyperspectral Geoscience Products," Proceedings of the International Geoscience and Remote Sensing Symposium (IGARSS'2006), Denver, Colorado, July 2006

[22] Neville, R.A., K. Staenz, T. Szeredi, J. Lefebvre, and P. Hauff, "Automatic Endmember Extraction from Hyperspectral Data for Mineral Exploration," Proceedings of the Fourth Int. Airborne Remote Sensing Conference and Exhibition and the $21^{\text {st }}$ Symposium on Remote Sensing, Ottawa, Ontario, Canada, Vol. II, pp. 891-897, 1999. 